


Review Article

\title{
Natural Super-Disintegrant Agents Used in Various Oral Solid Dosage Forms
}

\author{
V.T. Iswariya*, Nambaaru Sailaja, CH. Vamsi Krishna, G.S. Annammadevi \\ Department of Pharmaceutics, GITAM Institute of Pharmacy, GITAM, Visakhapatnam-500045, India
}

Article Info:
Article History:
Received 28 Oct 2020;
Review Completed 29 Dec 2020
Accepted 10 Jan 2021;
Available online 21 Jan 2021

Cite this article as:

Iswariya VT, Sailaja N, Krishna CV, Annammadevi GS, Natural Super-Disintegrant Agents Used in Various Oral Solid Dosage Forms, Journal of Drug Delivery and Therapeutics. 2021; 11(1):110-113 DOI: http://dx.doi.org/10.22270/jddt.v11i1.4681

\section{Abstract}

Super-disintegrating agents are one of the ingredients used in pharmaceutical solid dosage forms. These substances play a vital role in formulation design. Natural superdisintegrants have gained more popularity due to their oral bioavailability. It disintegrates the tablets into smaller particles to enhance the dissolution rate. Fast dissolving, chewable tablets, and other orally administered dosage forms consist of super-disintegrating agents which shows rapid and quick action. Natural super disintegrating agents in pharmaceutical dosage forms are very effective due to their ecofriendly nature as well as these are biocompatible and biodegradable. These are abundantly and cheaply available from nature.Today the researchers are focusing on naturally available excipients.

Keywords: Natural Superdisintegrants, Disintegration, Bioavailability, Biocompatible, Biodegradable.

\footnotetext{
*Address for Correspondence:

V.T. Iswariya, Department of Pharmaceutics, GITAM Institute of Pharmacy, GITAM, Visakhapatnam-500045, India
}

\section{Introduction}

Oral solid dosage forms consists of active pharmaceutical ingredients(having therapeutic activity) and excipients such as diluents, binders, disintegrantes, etc., which helps for delivery of drug and release at target site to show its effect.Superdisintegrant facilitates the breakdown of the tablets in the buccal cavity without any complexity of swallowing in the presence of saliva within a second. The term superdisintegrant, as its name proposes superior to disintegrate. Superdisintegrant is the substances that facilitate the lowering of the disintegration time (DT) even at low concentration, typically $1-10 \%$ by weight relative to the total weight of the dosage unit.1-3

Natural superdisintegrants includes gums (locust bean, chitin and chitosan), mucilage (isapghula husk, cress, gum karaya, fenugreek seed) and powders (agar, alginates, soy, polysaccharide, cassia fistula, xanthan, cucurbita maxima pulp, Hibiscus, mango peel pectin). ${ }^{4-7}$

\subsection{Advantages of Natural superdisintegrants:}

$\checkmark$ Easily available

$\checkmark$ Economic

$\checkmark$ Biocompatibility \& biodegradability

$\checkmark$ Increases patient compliance

$\checkmark$ Non-irritant and non-toxic

$\checkmark$ offer secure and effective drug delivery systems in the patients.

\subsection{Disintegration of oral solid dosage forms based upon}

$\checkmark$ Quantity of superdisintegrant subjected in the formulation.

$\checkmark$ Methods involved in the addition of superdisintegrant

$\checkmark$ Compatibility of superdisintegrants with drug and other additives. 


\subsection{Methods involved in the addition of superdisintegrants:}

Methods
-During granulation/
Intragranular/ internal
addition
-Prior to compression/
Extragranular/ External
addition
-Partially internal and
external
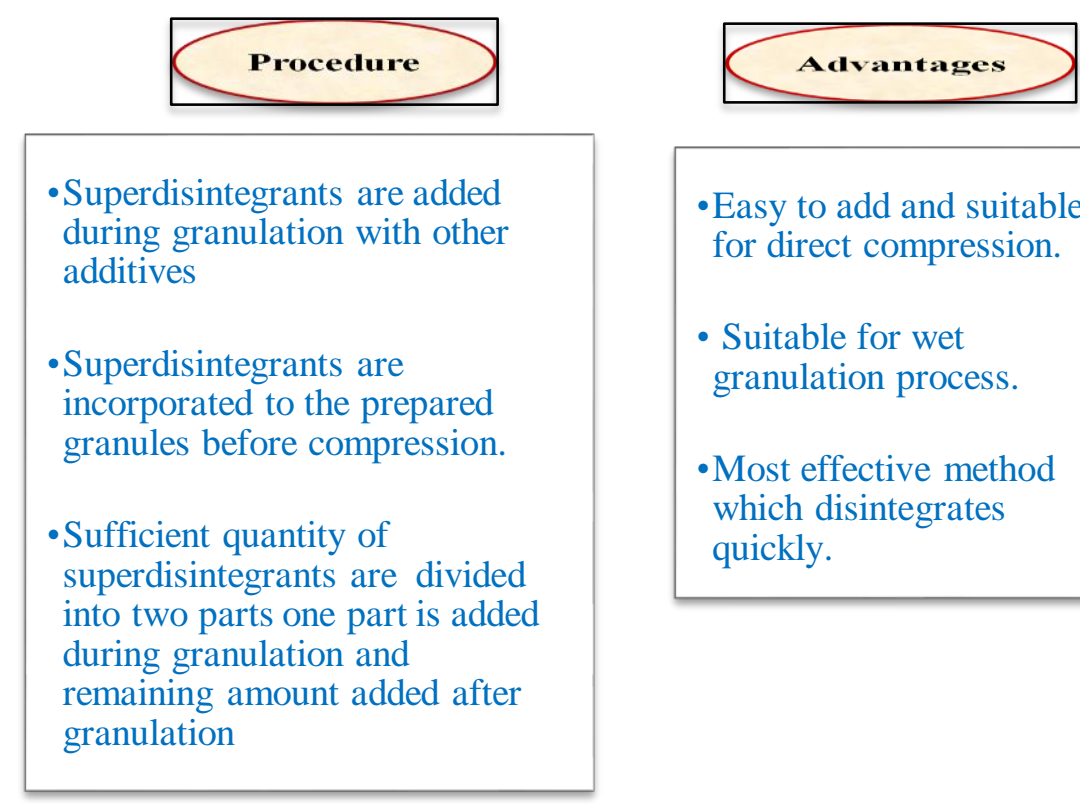

- Easy to add and suitable for direct compression.

- Suitable for wet granulation process.

- Most effective method which disintegrates quickly.

Scheme 1: Advantages and procedures of methods used for superdisintegrants

\subsection{Mechanism of action:}

The mechanism through which superdisintegrants facilitate quick breakdown of tablets into small fragments which results in faster dissolution and rapid onset of action are: 7

\section{Mechanism of action}

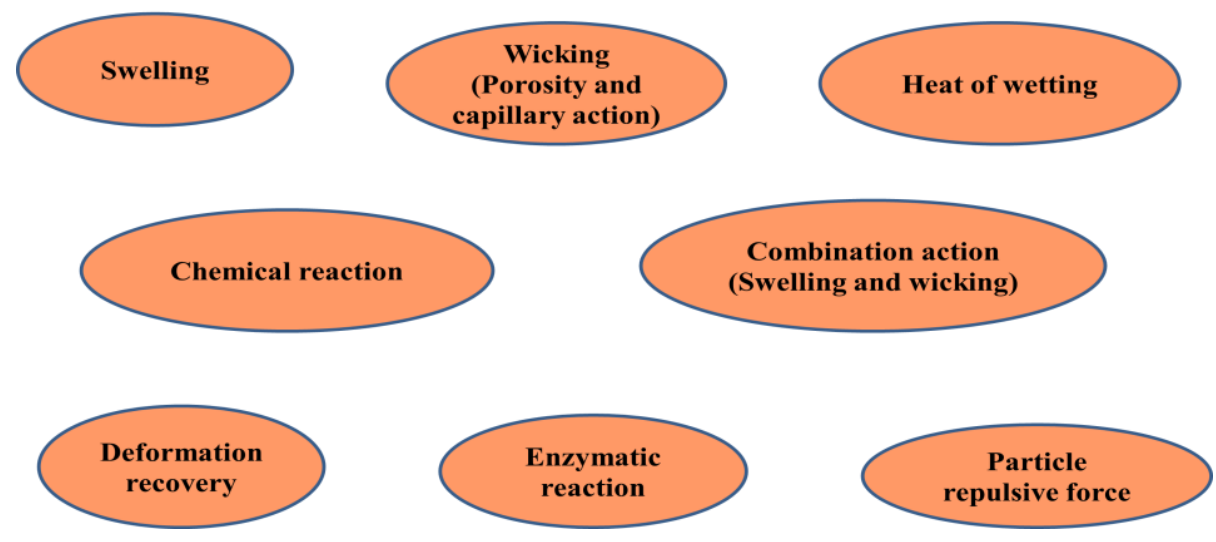

Scheme 2: mechanism of action

\section{i) Swelling}

Superdisintegrants common mechanism of action is swelling which disintegrates the tablets when it contact with suitable medium. The primary step involved in the mechanism is water penetration followed by swelling of the disintegrant particle which results in the breakdown of the tablet. ${ }^{8}$
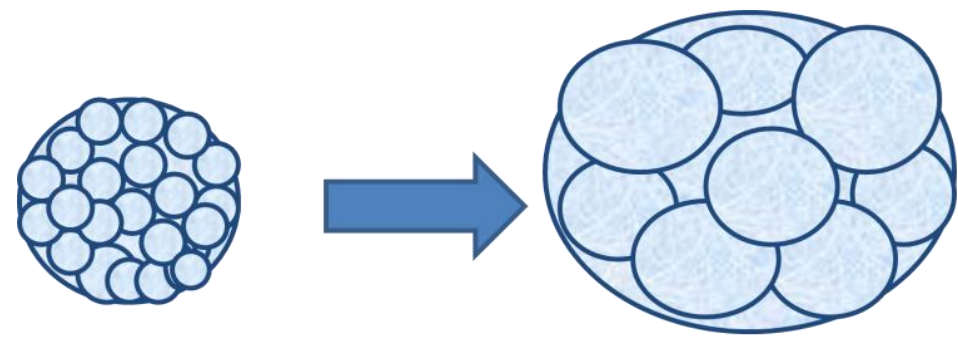
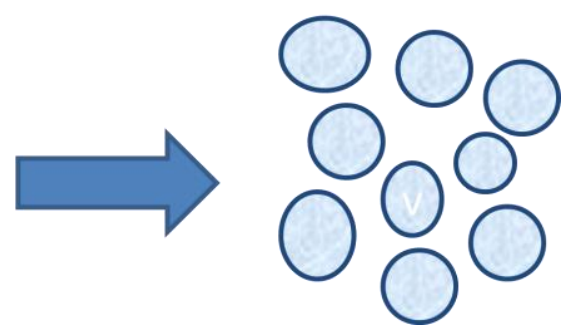

Figure 1: Swelling mechanism of tablet disintegration 


\section{ii) Wicking (Porosity and capillary action)}

Disintegration of tablet occurs by penetration of medium by replacing the adsorbed air on the particles results in weakening of intermolecular bond and breakdown of tablets into its fine particles. Water uptake of tablet is determined by hydrophilic property of the drug and excipient along with tableting conditions. For creating a hydrophilic network across drug particles, involves maintenance of porous structure and low interfacial tension towards aqueous fluid is a very crucial step in this mechanism. ${ }^{9}$

\section{iii. Heat of wetting}

Heat of wetting mechanism is applicable for the disintegrant having exothermic property. When these disintegrant comes in contact with suitable media and get wetted, there is a capillary air expansion leads to localized stress which causes disintegration of tablet.

\section{iv. Chemical reaction (Acid-base reaction)/Due to release of gases}

Disintegration mainly due to generation of pressure within tablet because of liberation of carbon dioxide from water, which forms when tartaric acid or citric acid reacts with alkali bicarbonates or carbonates (acid reacts with bases). Dissolution of active ingredient and excipients such as taste masking agents are also getting enhanced due to liberation of carbon dioxide gas. Environmental conditions should be strictly monitored as these disintegrant are highly sensitive to small change in temperature and humidity.

\section{v. Particle repulsive force}

Particle repulsive force mechanism is common in non swellable disintegrant particles leads to tablet breakdown. Tablet disintegration occurs due to electric repulsion between the particles and water is required for it. Particle repulsive theory which was proposed by Guyot-Hermann. It was found by researcher that repulsion is secondary to wicking. Guyot-Hermann repulsion theory proposed that, "Tablet in contact with suitable medium, water penetrates into the tablet through hydrophilic pores leading to the formation of continuous starch like network which helps in transfer of water from one particle to another and creates hydrostatic pressure." Thus, results in breaking of hydrogen bonds and other forces which hold tablet particles together.

\section{vi. Deformation recovery}

Deformation recovery mainly based on the principle that, some disintegrant particles structure get distorted or change while compression and after compression when it comes in contact with aqueous media, it returns to their precompression structure leading to the disintegration of the tablet. For example, starch has shown increased swelling capacity after the granules are compressed. High elastic nature of starch (like potato starch and corn starch) deformed to plasticity due to high compaction force while compression with energy rich potential. When these deformed starch particles comes in contact with water, tablet disintegration occurs as it triggers the energy rich potential of the deformed starch.

\section{vii. Enzymatic reaction}

Tablet disintegration occurs by some enzymes such as Amylase, Protease, cellulase and Invertase present in our body which acts as disintegrantes by decreasing the binding ability of the binder. Swelling cause pressure in the outer direction makes tablet to burst or more water absorption results in excessive granular volume promotes in disintegration of tablet.

\section{viii. Combination action}

Here, disintegrantes cause breakdown of tablet by combination of both swelling and wicking mechanism. Example: Crospovidone acts by combination of swelling and wicking.

\subsection{Super-disintegrants obtained from natural sources:}

\section{i.Lepidium Sativum-seeds:}

Lepidium Sativum herb used in most herbal formulations. It is also known as cress which belongs to family Cruciferae. It contains mucilage which acts as binding and disintegrating agents mainly based on swelling mechanism. Mostly the seeds contain high mucilage content compare to leaves. ${ }^{10}$

\section{ii. Isapghula husk/seeds:}

It is obtained from Plantago Ovata plant belongs to family plantaginaceae. The plant contains mucilage in the epidermis of the seeds. Mucilage acts as superdisintegranting agent in fast dissolving tablets due to their swelling property.

\section{iii. Fenugreek:}

It is obtained from Trigonella foenum-graceum herb belongs to family Leguminosae. It is widely used as food additive. Mucilage is an amorphous powder that quickly dissolves in warm water to form viscous colloidal solution.In fenugreek seeds high mucilage content was found so it is used as disintegranting agent in oral dissolving tablets.

\section{iv. Guar Gum:}

Guar gum extracted from the endosperm of the seed of the legume plant Cyamopsis tetragonolobus (family Leguminosae). Guar gum is prepared by firstdrying the pods in sunlight, then manually separating from the seeds. Chemically, guar gum is a polysaccharide composed of the sugars galactose and mannose. The backbone is a linear chain of 1, 4-linked mannose residues to which galactose residues are 1, 6-linked at everysecond mannose,forming shortside-branches. It mainly consisting of the high molecular weight (approximately 50,000- 8,000,000) polysaccharides composed of galactomannans. It is used as thickener, stabilizer and emulsifier, and approved in most areas of the world (e.g. EU, USA, Japan, and Australia). It is naturally occurring gum (marketed under the trade name jaguar). It is free flowing, completely soluble, neutral polymer composed of sugar units and is approved for use in food.

\section{v.Banana powder}

Banana synonym is plantain and belongs to the FamilyMusaceae. It contains retinol (Vitamin A). It also contains pyridoxal (Vitamin B6), which is given for reducing stress. It has rich source of carbohydrate and potassium which are responsible for brain functioning. In geriatric patients use of natural banana powder as a superdisintegrant in a formulation of drugs is more promising with respect to economical and compatibility.

\section{vi. Locust Bean gum}

Locust bean gum also called Carob bean gum. It is extracted from the endosperm of the seeds of the carob tree Ceratonia siliqua, which grows in Mediterranean countries. Some other familiar polysacharides are starch and cellulose, which are made of long chains of the sugar glucose. In locust bean gum, the ratio of mannose to galactose is higher than in guar gum, giving it slightly different properties, and allowing the two gums to interact synergistically so that together they make a thicker gel than either one alone. It shows as a binder and as 
a disintegrant property at different concentration. Locust bean gum has been widely used in food industry as a thickening and gelling agent. Locust bean gum has also been reported to have bioadhesive and solubility enhancement properties.

\section{vii. Mango peel pectin}

It is obtained from magnifera indica plant belonging to Anacardiaceae family. Mango peel which constitutes 20$25 \%$ of the mango processing waste was found to be a good source for the extraction of pectin of good quality, suitable for the preparation of film and acceptable jelly. Pectin is a complex hetro-polysacharides which is a hydrophilic colloid. Due to its good solubility and higher swelling index, it may be used in the formulation of fast dispersible tablets.

\section{vii. Agar and treated agar}

Agar is the dried gelatinous substance obtained from Gelidium amansii (Gelidanceae) and several other species of red algae like Gracilaria (Gracilariaceae) and Pterocadia (Gelidaceae). Agar is yellowish gray or white to nearly colorless, odorless with mucilaginous taste and is available in the form of strips, sheet flakes or coarse powder. Agar consists of two polysaccharides as agarose and agaropectin. Agarose is responsible for gel strength and Agaropectin is responsible for the viscosity of agar solutions. High gel strength of agar makes it a potential candidate as a disintegrant.

\section{viii. Gellan gum}

Gellan gum is a water-soluble polysaccharide produced by Pseudomonas elodea, a bacterium. Gellan gum is an anionic, high molecular weight, deacetylated exocellular polysaccharide gum produced as a fermentation product by a pure culture of Pseudomonas elodea2, with a tetrasaccharide repeating unit of one $\alpha$-L-rhamnose, one $\beta$ $D$-glucuronic acid and two $\beta$-D-glucose residues. Several studies were conducted on Gellan gum as a disintegrant and the efficiency of gum was compared with other conventional disintegrants such as dried corn starch, explotab, avicel $\mathrm{pH}$ 10.2), Ac-di-sol. and Kollidon CL. The disintegration of tablet might be due to the instantaneous swelling characteristics of gellan gum when it comes into contact with water and owing to its high hydrophilic nature. ${ }^{11,12}$

\section{ix. Soy polysaccharide}

It is a natural superdisintegrant that does not contain any starch or sugar so can be used in nutritional products. soy polysaccharide (a group of high molecular weight polysaccharides obtained from soy beans) as a disintegrant in tablets made by direct compression using lactose and dicalcium phosphate dihydrate as fillers. A cross- linked sodium carboxy-methyl cellulose and corn starch were used as control disintegrants. Soy polysacchardie performs well as a disintegrating agent in direct compression formulations with results paralleling those of cross-linked CMC. ${ }^{13}$

\section{x. Chitin and chitosan}

Chitin $(\beta-(1 \rightarrow 4)-\mathrm{N}$-acetyl-D-glucosamine $)$ is a natural polysaccharide obtained from crab and shrimp shells. It possesses amino group covalently linked to acetyl group as compared to free amino group in chitosan. Chitosan is produced commercially by deacetylation of chitin, which is the structural element in the exoskeleton of crustaceans (such as crabs and shrimp) and cell walls of fungi. The reported data showed that when chitin was included in the conventional tablets, the tablets disintegrated with in 5 and 10 minutes irrespective of solubility of the drug. The disintegration time in the oral cavity as well as wetting time could be analyzed by surface free energy. Chitosan is the best known natural polysaccharide used for its versatile applications in pharmaceutical industry.

\section{xi. Gum Karaya}

Gum Karaya is a vegetable gum produced as an exudate by trees of the genus Sterculia. Chemically, Gum Karaya is an acid polysaccharide composed of the sugars galactose, rhamnose and galacturonic acid. The high viscosity nature of gum limits its uses as binder and disintegrant in the development of conventional dosage form. Karaya gum has been investigated for its potential as a tablet disintegrant. Various results showed that modified Gum Karaya produce rapid disintegration of tablets. Gum Karaya can be used as an alternative superdisintegrants to commonly available synthetic and semisynthetic superdisintegrants due to their low cost, biocompatibility as well as easily availability.

\section{References}

1. Pratik R.A, Hitesh P, Sunita A.C, et al., Review article Inventi Journals.2014; 3.

2. Guptha D.K, Tyagi.S et al International Journal of Advanced Research, 2013; 1:576-583.

3. Gandhi L, Akhtar S, Journal of Drug Delivery \& Therapeutics. 2019; 9(2):507-513.

4. Shivprasad H.M et al. Asian Journal of Biomedical and Pharmaceutical Sciences. 2012; 2:69-72.

5. Pahwa and Gupta. IJPSR, 2011; 2:2767-2780.

6. Kushagra Khanna et al. Int.J.Pharm.Res. Allied Sci.,2016; 5:311322.

7. Mohanachandran P.S,Sindhumol P.G et al International Journal of Pharmaceutical Sciences Review and Research.2011; 6.

8. Vikas Sharma,Vandana Arora, et al. International Bulletin of Drug Research.1,46-54.

9. Santosh Kumar R, Kumari A. Journal of Drug Delivery \& Therapeutics. 2019; 9(2):461-468.

10. Rawat S, Derle DV, et al. World journal of pharmacy and pharmaceutical sciences. 2014; 3:263-278.

11. Shirsand SB, Jonathan V, et al. Indo American Journal of Pharmaceutical Sciences, 2015; 3:275-282.

12. Sujatha K.M, Rajesh K, Kishore B.M, et al. International Journal of Pharmacy \& Pharmaceutical Research. 2019; 17:187-210.

13. Tausif Alam M D, Nayyar Parvez , et al. Journal of Pharmaceutics. 2014; 6. 